\title{
Terahertz microscopy with oblique subwavelength illumination in near field
}

\author{
Oleg V. Minin ${ }^{1}$, Igor V. Minin ${ }^{1, *}$ \\ ${ }^{1}$ Tomsk Polytechnical University, \\ 30 Lenin Ave., Tomsk, 634050, Russia \\ *Email:prof.minin@gmail.com
}

\begin{abstract}
Microscopes based on dielectric mesoscale particles, using the effect of a photonic jet or terajet in the terahertz range, are a promising tool for overcoming the diffraction limit. However, the image they generate has limited contrast, which limits the application of this method. In this letter, we demonstrate that it is possible to increase the contrast of an image based on dielectric mesoscale particles that provide the formation of photonic hooks. In this case, the illumination of the object is carried out by an oblique incidence of subwavelength terajet, which significantly (more than 2 times) increases the contrast of the image.
\end{abstract}

Key words: photonic hook, terajet, oblique illumination.

Introduction. Conventional optical microscopes cannot resolve two objects closer than $0.5 \lambda / \mathrm{nNA}$, where $\lambda$ is the incident wavelength, NA is the numerical aperture of the microscope lens, $\mathrm{n}$ is the refractive index of the immersion medium, due to the Abbe diffraction limit. In 2017, it was shown that to increase the spatial resolution of terahertz (THz) microscopes by simply placing a dielectric mesoscale (comparable to the wavelength) particle in the focal area of the optical system [1], which forms a terajet with a subwavelength transverse size of the focal area. On the other hand, it is known that oblique illumination makes it possible to improve the contrast of an image [2] by capturing diffraction fields of higher orders.

In this work, we propose the concept of a $\mathrm{THz}$ microscope that combines these two approaches: the use of oblique illumination of an object using structured subwavelength fields in the near field in the form of terajet and / or a photonic hook (PH) [3].

Concept. The key problem in this concept is the principle of a particle design that will ensure the formation of a terajet formed at an angle to the direction of incidence of radiation (Fig. 1). In the case of axial terajet formation, the diffracted orders -1 and +1 of the radiation reflected from the object are usually outside the particle boundary (Fig. 1a). Oblique illumination leads to the fact that the diffracted +1 order falls on the particle (Fig. 1b), which ultimately makes it possible to increase the image contrast [2].

In [4], a simple method for generating a $\mathrm{PH}$ using a dielectric cubic particle, along the side face of which a metal screen is installed [4] was proposed. PH generation is based on the control of the tangential component of the electric field along the particle side surface [4].

Simulations. The problem was simulated within the framework of the numerical solution of Maxwell's equations using the CST Microwave Studio ${ }^{\mathrm{TM}}$ software with a minimum mesh size of $\lambda /(25 n)$, where $\mathrm{n}=1.46$ for Teflon. As in [1], the cube, immersed in vacuum, has dimensions equal to the wavelength $(\lambda)$ and is illuminated with a vertically polarized plane wave at $0.1 \mathrm{THz}$.

Discussions and results. Figure 2(a) shows the formation of a photonic hook, the optical path of which is displaced from the symmetry axis and curved due to the side metal screen. The localized photonic flux formed near the shadow surface of the particle is directed at an angle to the optical axis $(\alpha=$ $24^{0}$ ), which allows oblique illumination of the object. 


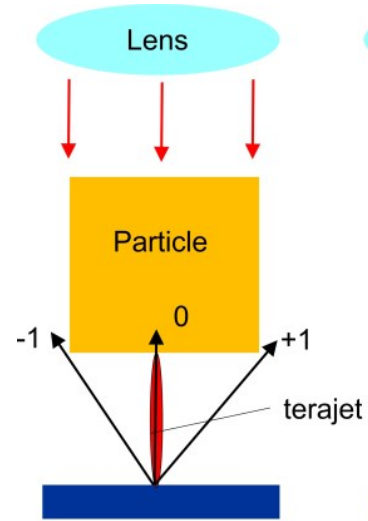

a)

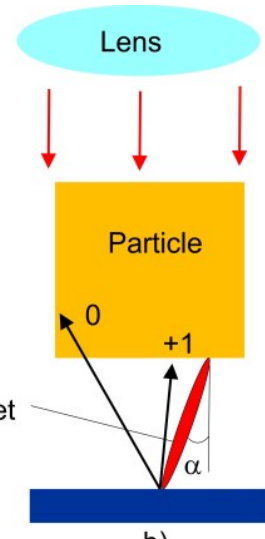

b)
Fig. 1. The concept of a microscope with a particle forming a terajet (a) and with oblique illumination with a terajet or $\mathrm{PH}(\mathrm{b})$. The numbers at the arrows indicate the diffraction order, $\alpha$ is the angle between the terajet axis and the optical axis.

The scheme and parameters of the experiment were similar to Ref.[1]. As a sample object, metallic plate with $0.63 \lambda$ trenches was used [1]. The contrast of the image increased by about 2.2 times, as shown in Figure 2(b), due to the use of oblique illumination with a photonic hook instead of normal illumination with a terajet. The image contrast was defined as $\left(I_{\max }-I_{\min }\right) /\left(I_{\max }+I_{\min }\right)$, where $I_{\min }$ and $I_{\max }$ are the minimum and maximum detected amplitudes, respectively [1].
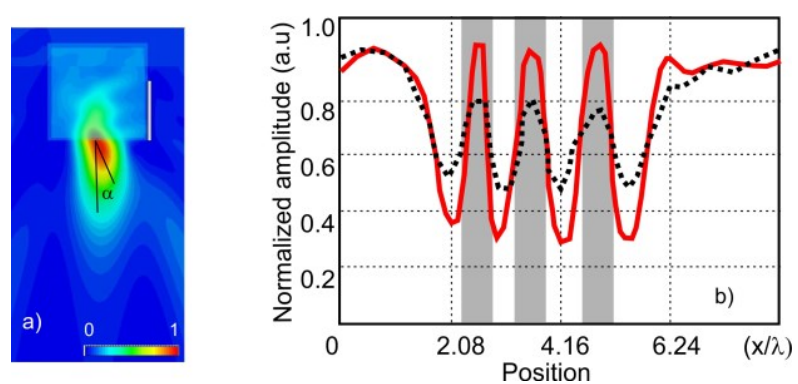

Fig. 2. (a) - formation of a photonic hook by a cubic particle with a side screen, (b) - experimental contrast of the image of a test object with a terajet (dotted line) and a photonic hook (solid red line).

Conclusion. This article shows the fundamental possibility of creating an oblique illumination microscope in the near field, which makes it possible to increase the image contrast and has an ultra-high resolution, inherent in terajet, without using an immersion medium (liquid). Mesoscale dielectric particles can generate a photonic hook due to their different structural asymmetry or asymmetry of the illuminating wavefront [3]. Optimization of spatial resolution is achieved by a reasonable compromise between particle size, shape, optical contrast and angle of oblique illumination.

\section{CRediT authorship contribution statement Oleg V. Minin: Conceptualization, Formal} analysis, Investigation, Data curation. Igor V. Minin: Writing - review \& editing, Supervision. This research was partially supported by TPU development program.

\section{Declaration of Competing Interest}

The authors declare that they have no known competing financial interests or personal relationships that could have appeared to influence the work reported in this paper.

\section{References}

1. H. H. N. Pham, S. Hisatake, O. V. Minin, T. Nagatsuma, and I. V. Minin. Enhancement of spatial resolution of terahertz imaging systems based on terajet generation by dielectric cube. APL Photonics 2, 056106 (2017); DOI: 10.1063/1.4983114

2. J.Price, P.Bingham, and E.Thomas, Improving resolution in microscopic holography by computationally fusing multiple, obliquely illuminated object waves in the Fourier domain. Applied Optics 46(6), 827-833 (2007); DOI: 10.1364/AO.46.000827

3. O. V. Minin, I. V. Minin. The Photonic Hook: From Optics to Acoustics and Plasmonics. (Springer, Cham, 2021); DOI: 10.1007/978-3-030-66945-4

4. I.Dorofeev, V. Suslyaev, I.V. Minin, O.V. Minin. The role of the tangential electric field component to the terahertz jet and hook formation by dielectric cube and sphere. Opt Eng 60(8), 082004 (2020); DOI: 10.1117/1.OE.60.8.082004 